\section{La complejidad común De la composición arquitectónica y musical}

\section{Josep Llorca}

"Lo «real» que hay en la obra de arte, las superficies y masas, los colores y materiales, los sonidos con sus leyes armónicas; todo eso tiene carácter de indicación por la cual el artista se pone de acuerdo con el contemplador sobre lo que realmente pretende. Esto se encuentra en ese espacio irreal que el hombre logra abrir mediante su mirada e imaginación, y desde el cual se pone en tensión hacia la realidad. Naturalmente, no se puede separar de lo real exterior, sino que está unido a ello, forma con ello esa unidad característica que precisamente se llama "obra de arte»."

T ratar de explicar lo que uno mismo siente ante una obra musical y arquitectónica y justificar su punto de vista en esa explicación no es tarea fácil ni, quizá, útil; puede servir para que otros que se encuentran en el mismo trance se vean identificados o, por el contrario, para que lo tachen a uno de loco. Esto es justamente lo que se me pasa por la cabeza cada vez que retomo este ensayo sobre dos temas que, a mi parecer, están estrechamente ligados: la composición arquitectónica y la composición musical. No obstante, creo que es importante hablar de ellos con insistencia porque no están ligados de una forma superficial, sino que comparten en sus bases una misma lógica de funcionamiento: la sucesión y ensamblaje de piezas para conseguir una atmósfera. La manera de organizar piezas para conseguir una atmósfera. La manera de organizar a", es decir, los objetos son ellos mismos en y a través de los otros y por tanto se pueden extraer conclusiones que ayudan a comprender mejor ambas disciplinas. Los arquitectos buscan incesantemente la manera de construir un lugar exquisito que comprenda la complejidad del hombre y la relación con el encomprenda la complejidad del hombre y la relación con el entorno. Los músicos, por su parte, tratan de ofrecer al hombre hombre se identifique. Los dos caminan hacia un mismo lugar hombre se identifique. Los dos caminan hacia un mismo lugar
muchas veces sin saberlo. Percatarse de este dato puede ser muchas veces sin saberlo. Percatarse de este dato puede ser
revelador a la hora de resolver cuestiones que atañen a la esenrevelador a la hora de resolver cuestiones que atañen a la esen-
cia de ambas disciplinas, intentando buscar siempre lo que es auténtico y peculiar de cada una de ellas.

\section{La atmósfera habitable}

A menudo se difunde la idea de que la música y la arquitectura comparten aspectos como el ritmo, la armonía entre las partes y el todo, el manejo de las proporciones o los contrastes. Estas ideas no son falsas, aunque tampoco terminan de ser completas porque podrían realizarse en comparación con otras muchas disciplinas sin tener en cuenta el núcleo duro que las une. Lo que de verdad las distingue de las demás es que solamente la música y la arquitectura son capaces de crear un hábitat para que el hombre viva. Eugenio Trías lo expresa con las siguientes palabras en su Lógica del Límite:

"En ambas, música y arquitectura, se sitúa en primer plano, a diferencia de otras artes (pintura, escultura, artes del lenguaje) la dimensión del habitar. La música, lo mismo que la arquitectura, debe ser habitada. Eso significa que mantienen ambas un nexo inmediato y espontáneo con el hábitat (Umwelt, ambiente). La música "nos envuelve", como en general toda la sonoridadambiente, del mismo modo como "nos envuelve" el ámbito que determina la arquitectura. Crea una segunda naturaleza (ya formada) en relación con la primera, salvaje y sin cultivar .

Y esta segunda naturaleza o hábitat acaba conformando lo que los arquitectos o músicos llamamos atmósfera, el medio característico que cada obra en particular ofrece al individuo. Y la llamamos atmósfera porque la música y la arquitectura no tienen una carga semántica, son abstractas y, por consiguiente constituyen el limite entre el hombre y la naturaleza salvaje que lo rodea, intermediarias entre el individuo y la cultura. Ambas crean un lapso de tiempo o de espacio en el que se tensa el aire y dan como fruto la atmósfera: tensar el aire con materia y luz o tensar el aire con sonidos y silencios.

Peter Zumthor ha insistido en el concepto de atmósfera defendiendo que la calidad arquitectónica de un edificio sólo puede provenir de que conmueva o no, de la atmósfera, y ejemplifica este hecho con una obra musical:

En el primer movimiento de la sonata para viola de Brahms (sonata n 2 en mi bemol mayor para viola y piano), cuando entra la viola, en un par de segundos ya está ahi, y no sé bien porque. Y algo parecido ocurre también en arquitectura. No tan poderosamente como en la más grande de las artes, la música, pero también está ahí ${ }^{3}$

Todos los demás aspectos que pudieran relacionar a las dos disciplinas se someten a este fin, el de la creación de la atmósfera. Por ejemplo, si nos colocamos en el proceso de gestación de una pieza vemos que, aunque los proyectos de arquitectura se manejen mediante vistas, planos y maquetas -elementos todos ellos de aproximacion a la realidad- y la obra musical se geste en la partitura -elemento también de aproximación a la realidad-, ambas sólo cobran sentido cuando se ejecutan, cuando se ponen en obra. De aquí que sea tan importante el proceso de ejecución: la prueba de fuego de la que resultará una atmósfera u otra diferente.

Por eso trato de descifrar cómo a pesar de sus enormes diferencias de material base -espacio y tiempo- ambas consiguen imprimir en nosotros sensaciones de ambientes o atmosteras mediante unos mecanismos que, pudiendo clasificarse tanto en estructurales como fenomenológicos, no están reñidos entre sí. Es más, precisamente en la genial articulación entre las estructuras formales y el control de su percepción en la persona radica que satisfaga sus necesidades.

\section{Las funciones formales}

El pensamiento estructuralista que Deleuze y sus discípulos impulsaron en la década de los 30 defendía la existencia de unas estructuras bajo el caos aparente de las cosas. Este pensamiento ha tenido una enorme resonancia tanto en la arquitectura como en la música, en los conceptos de "tipo" o "estructura formal" de las obras. En arquitectura, la crítica tipológica ${ }^{4}$ trata de descubrir el orden entre los espacios, su articulación o su jerarquia dentro del edificio a lo largo de la historia. Se habla de estructura sin referirse solamente a la estructura física, sino a la relación lógica entre espacios, que puede ser geométrica o topológica. En música, el análisis formal ${ }^{5}$ busca de igual manera el orden entre las funciones formales, sus articulaciones y su jerarquía dentro de la obra.

En este contexto, la obra de Louis Kahn y la del mismo Johann Sebastian Bach denotan la importancia de una estructura robusta que sustente el discurso de las obras. Aunque Kahn (19011975) ofrezca a la arquitectura del siglo XX una destilación de tipologías históricas y en la obra de Bach (1685-1750) culmine la riqueza formal y la capacidad expresiva de la música barroca, la riqueza formal y la capacidad expresiva de la música barroca, los elementos básicos de las estructuras formales. Los proyectos de Kahn demuestran la habilidad de articular formas puras -cuadrados, triángulos, círculos- mediante lógicas o sistemas -cuadrados, triangulos, circulos- mediante logicas o sistemas la simetría simple y múltiple, la inscripción de figuras dentro de la simetria simple y múliple, la inscripción de figuras dentro de otras, la articulación entorno a un claustro o la suma de edificios heterogéneos. Estas formas de articulación responden a la necesidad de jerarquizar los espacios según sus funciones obedeciendo a la distinción kahntiana entre "espacios servidos" y "espacios servidores". Del mismo modo, la fuga, joya del legado de
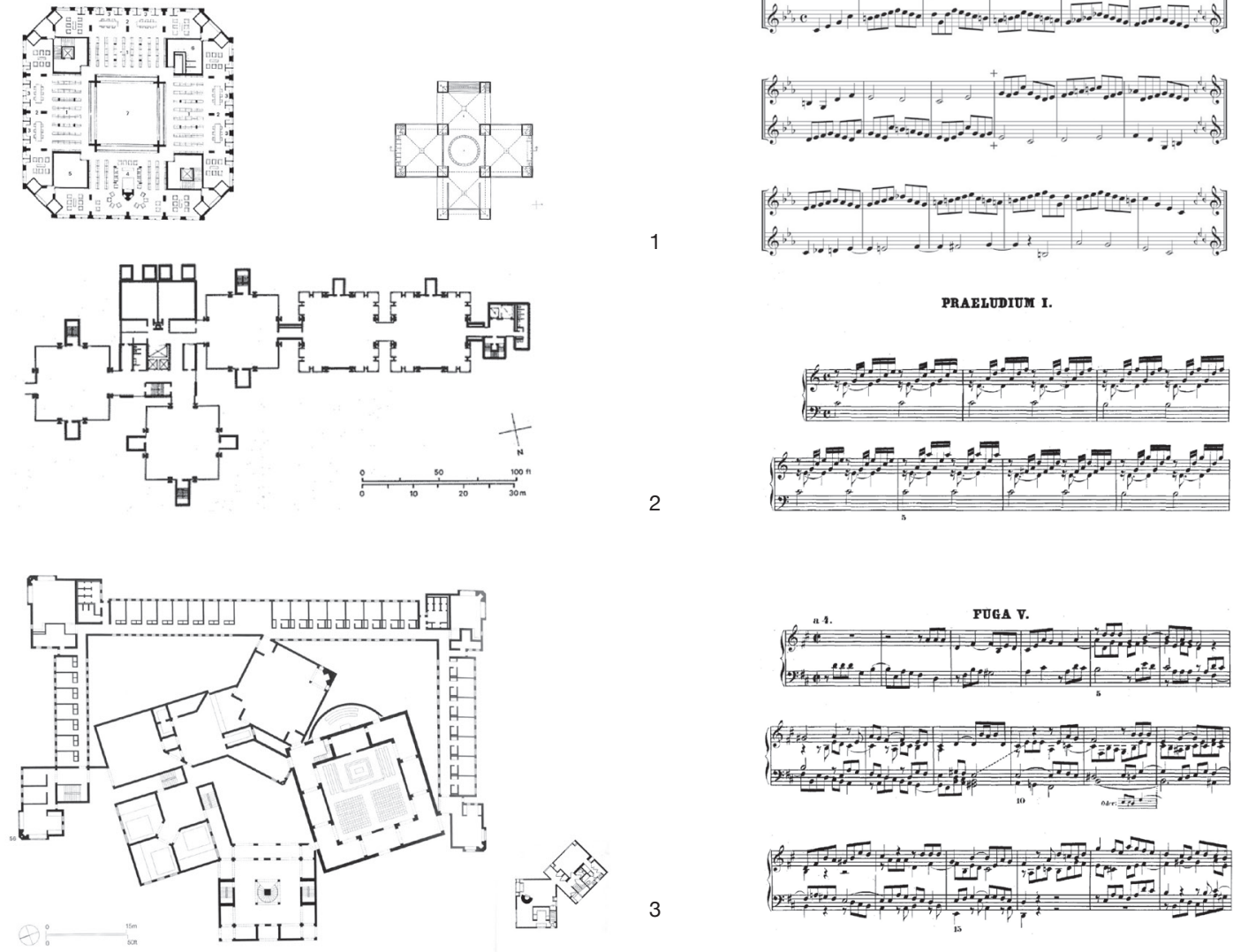
(6+a)

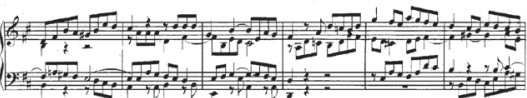

(1)
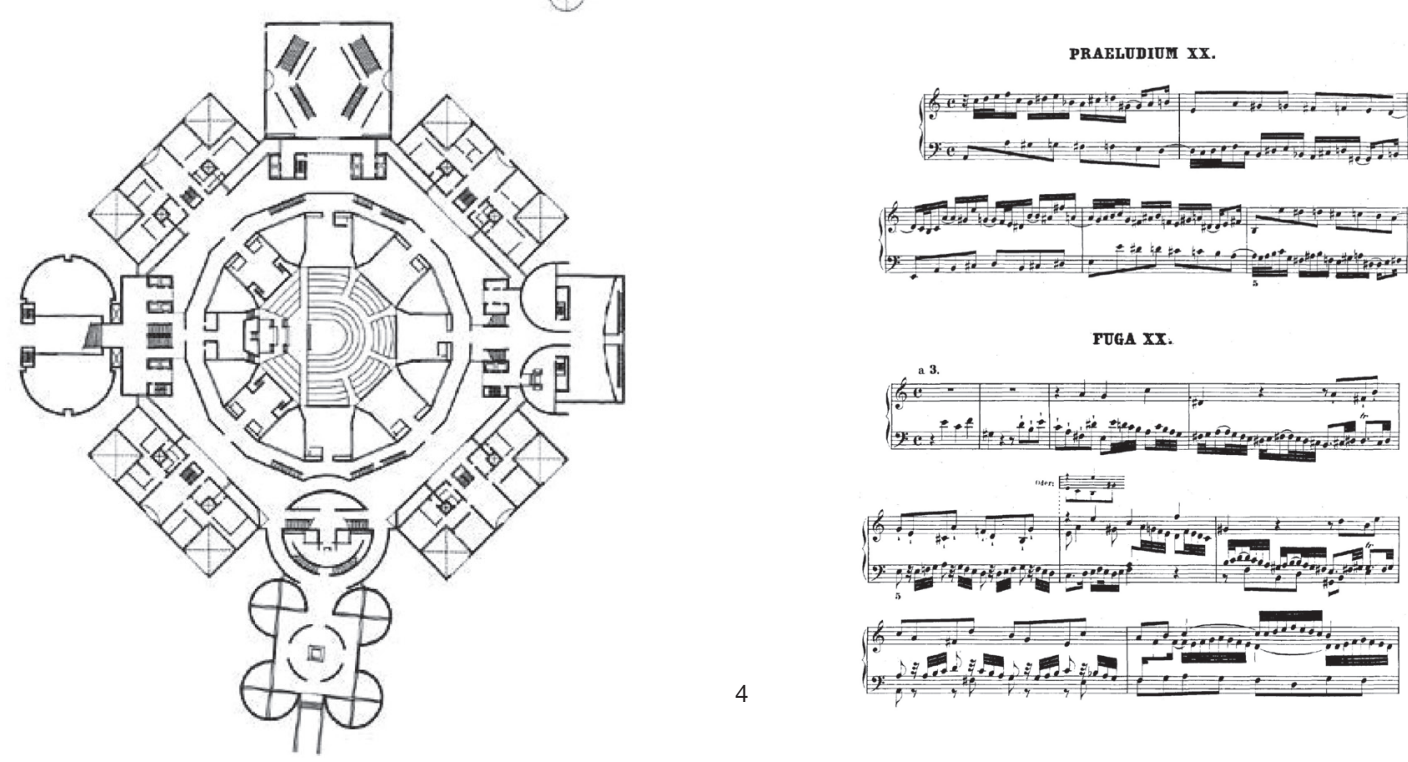

PUGA Xx.

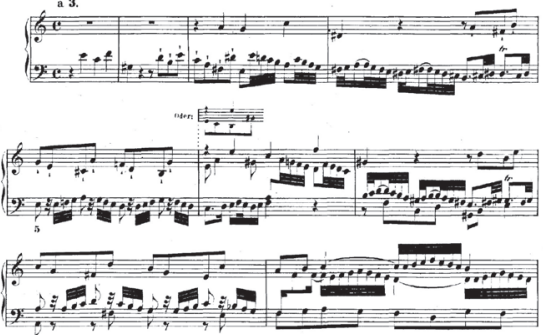

Fig. 1 Maneras de articular piezas para construir atmósferas en Kahn y Bach. 1: Simetría múltiple y contrapunto invertible en la biblioteca Exeter, los baños de Trenton y el Canon Cangrejo. 2: La repetición o agregación de piezas en los laboratorios Richards y el Preludio I del Clave Bien Temperado I. 3: La yuxtaposición o intersección de figuras simples en el convento de religiosas, la casa Fisher y la Fuga Asamblea de Dacca y el preludio y fuga XX del Clave Bien Temperado il. 
Podría parecer que el pensamiento estructuralista se estancase en conceptos que ya han sido tratados históricamente y que ofrecen poca novedad; sin embargo, la fuerza de una estructura robusta hace que la obra sobrevuele los gustos y las modas mo tiempo la hace atemporal. Los modos de articulación misBach investigó son los indicios Lora la form de articulación que Bach ins etc) institucionalizó y que hoy en día (Mozar, Haydn, Weber, son. ina forma minué forma rondó, etc. Estas formas no porma su vigen tructuras lógicas y una mirada atenta al panorama musical las reconoce sin problemas.

\section{Los fenómenos estructurales}

Sin embargo, valorar la obra de Kahn o Bach tan sólo por su habilidad estructural es insuficiente y no permite comprender su totalidad. Por eso, otros autores anteponen el papel de los sentidos (vista, tacto, oído, olfato y gusto) en la comprensión del mundo a la estructura mental. La atmósfera está, entonces, ligada a la percepción sensorial que nos ofrece la obra. Gaston Bachelard defiende que lo único que permanece en nosotros de un lugar es la sensación que tuvimos de él:

"¿De qué serviría dar el plano del cuarto que fue realmente mi cuarto, describir la pequeña habitación en el fondo de un granero, decir que desde la ventana, a través de la desgarradura de los tejados, se veía la colina? Yo solo, en mis recuerdos de otro siglo, puedo abrir la alacena profunda que conserva todavía, para mí solo, el aroma único, el olor de las uvas que se secan sobre el zarzo "9

Ígor Stravinsky describe el fenómeno musical como arte del tiempo, como una crononomía y, por tanto, defiende el trabajo con el tiempo como fundamento de las sensaciones producidas por la música:

¿Quién de nosotros, al escuchar una música de jazz, no ha experimentado una sensación divertida proxima al mareo, cuando ve que un bailarín o un músico solista que se obstina en marcar acentos irregulares no puede libertar su oído de la pulsación métrica regular mantenida por la percusión?

Toda la obra de Stravinsky es una oda a la poliritmia, una demostración del control de ritmos simultáneos y su impacto en el oyente. Al escuchar el inicio de la Danza de la tierra, de la Consagración de la primavera de 1913, constatamos ese poder en nosotros.

El análisis fenomenológico ${ }^{12}$ ha puesto énfasis en todos los aspectos sensoriales de la arquitectura organizándolos por capas: la textura de los materiales, el color, el olor, el sonido, los recorridos por el edificio y la geometría del espacio. La música, que trabaja sobre el sonido, también ha desgranado los elementos que percibimos por los sentidos en los conceptos de cadencia, tonalidad, tempo, métrica, ritmo, dinámica, densidad, timbre, registro, textura y motivo, entre otros, y los ha llamado "fenómenos estructurales".

El término "fenómenos estructurales" incluye perfectamente el universo de percepción dentro del de estructura. Y "las funciones formales" se concretizan en texturas, ritmos, colores, geometrías, cadencias, etc. Las funciones formales necesitan de los fenómenos estructurales para concretizarse y no existen formas si no se presentan como fenómenos. Esta conciencia de complementariedad proporciona a las obras una coherencia y accesibilidad indispensables para que el individuo las habite. Y sólo el arte del espacio y el arte del tiempo pueden ofrecer este obsequio a la humanidad.

\section{La complejidad enriquece}

Hasta aquí hemos comprendido que las obras musicales y arquitectónicas crean atmósferas gracias a todos los objetos reales que configuran los "fenómenos estructurales" y las "funciones formales". Sin embargo, una mirada atenta a la historia nos revela que hay autores que trabajan de una manera clara estos objetos reales y que el observador los percibe con rapidez y transparencia; se respira una atmósfera de serenidad y orden. Es el caso de las obras del periodo iluminista arquitectónico -los edificios ordenados de la escuela de Durand-, o las del período clásico musical -casos brillantes son Haydn o Mozart. Sin embargo, hay otros que entremezclan los límites entre las funciones y los fenómenos y consiguen crear ambientes más ambiguos entre sus partes, las relaciones se confunden y quedan superpuestas, aumenta la complejidad de la obra y, al mismo tiempo, la riqueza de relaciones. Estas atmósferas son características de la estética del romanticismo y el impresionismo musicales, las vanguardias de Xenakis, Ligheti y Stockhaussen o el indeterminismo de John Cage, donde las complejidades -a veces contradictorias- de relación entre las partes de la obra enriquecen el resultado. Pero también son características de las arquitecturas paisajísticas de Alvar Aalto o William Turnbull, las estrategias de fragmentos de Rem Koolhaas o las formas del caos de Daniel Libeskind, donde las complejidades -a veces contradictorias, de nuevo- del entorno de un proyecto enriquecen las relaciones entre las piezas. El poder atractivo de una atmósfera puede venir tanto de su serenidad, orden y equilibrio interno como de la riqueza, contraste y compleildad entre sus partes.

El caso de Debussy en el Preludio para la siesta de un fauno ilustra descendente de la flauta rompe el silencio de la sala y durante unos instantes permanece danzando por el aire hasta que una trompa acompañada por la orquesta responde al diálogo. La flauta toca por segunda vez el tema principal y, en vez de terminarlo a solas, alarga la última nota hasta fundirla con el sonido del oboe que recoge la misma nota y hace de ella el comienzo de lo que será el tercer motivo de la obra. Este pequeño fragmento encarna la atmósfera de toda la obra. Y lo podemos ver en el motivo principal, ( rítmica y su capacidad de amoldarse a cualquier su inestabilidad terier base armónica. Pero también lo podemos ver en la manera en que se relacionan (

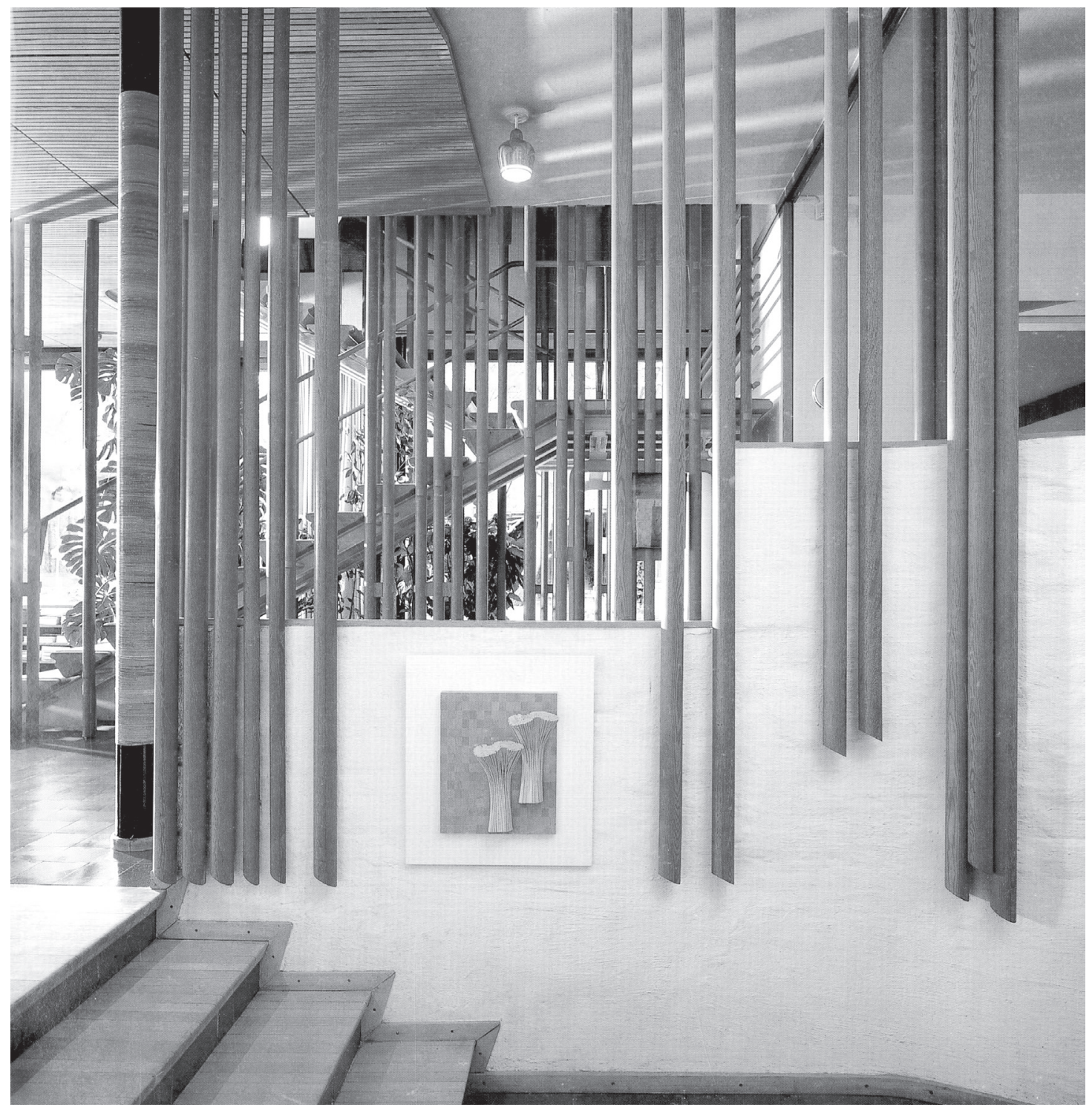

Fig. 2 Los espacios y sus funciones se funden en la Villla Mairea de Alvar Aalto. El observador puede recon
el salón a la izquierda, la escalera y el jardín al fondo en un solo golpe de vista. Fotografía: Rauno Träskelin

rastro de una melodía clara; tampoco podemos descubrir un ritmo constante ni repetitivo, sino más bien un conjunto de tempos que se deforman para dejar paso al siguiente.

\section{Conclusión}

La sociedad de hoy en día es muy propicia a valorar las obras tanto arquitectónicas como musicales tan sólo en su superficie cuando se podría extraer mucho más de ellas. El análisis formal, que hemos descrito anteriormente, es un modo de aproximación a las obras que no las seca, no las mata, sino que las descompone para descubrir sus partes y las recompone para admirarse de la totalidad. Sin embargo, la mirada fenomenológica ofrece el abanico de la percepción subjetiva, las sensaciones que a cada uno le producen las obras. Ambas son necesarias, pero resultan todavía insuficientes si queremos comprender la atmósfera. Es necesario vivirlas, enfrentarse con la realidad y empaparse de ellas para conocer el Ser mismo de cada obra, de su esencia, de toda la profundidad de su mensaje. Caminar la ciudad, recorrer los edificios, dormir en la habitación, entrar en el vestíbulo, escuchar el agua, perderse on la infinidad de timbres de una orquesta dejarse perpertir por el marill cutir por el martillo del piano, sentir el rozamiento de la cuerda de contrabajo, esperar el silencio entre dos movimientos o sumergirse en los ritmos desarticulados de una pieza de Jacques Loussier. La tanto, captar la atmósfera significa vivirlas.

"Pero eso lo logra [el contemplador] en cuanto que se esfuerza - y ya eso lo desconocen muchos, que sea necesario esforzarse, concentrarse, penetrar, aprender y ejercitarse, porque ven en la mientras que solo una cosa para horas de ocio, una "diversión", mientras que, por el contrario, pertenece al orden de las cosas altas, que presenta exigencias para poderse comunicar. El Pargrande esfuerzo como la filosofía de Platón."

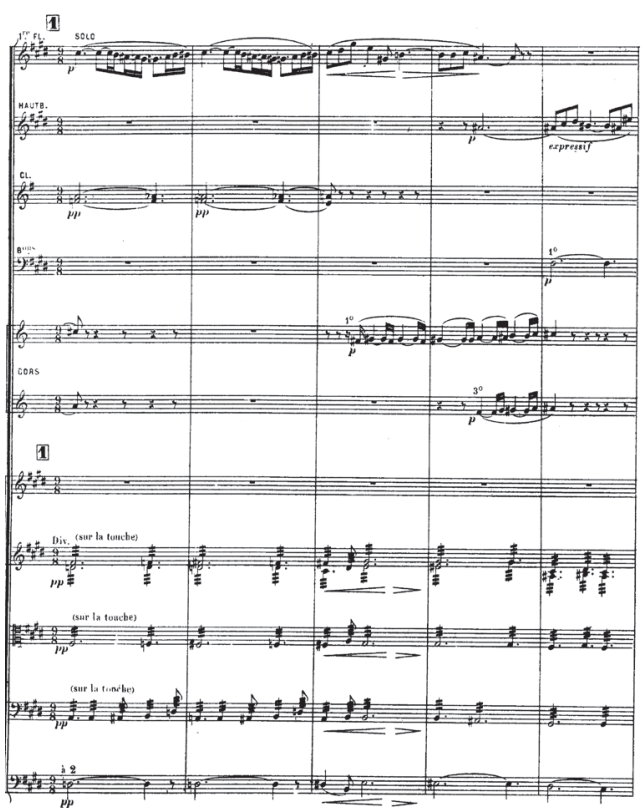

Fig. 3 Página del Preludio para la siesta de un fauno donde se funden el motivo de
en web: www.ismlp.org
GUARDINI, Romano, "Sobre la esencia de la obra de arte", en Imagen de
culto e imagen de devoción; Sobre la esencia de la obra de arte, Madrid, Guadarrama, 1960, p. 67

Eugenio, Logica del limite, Ediciones Destino, S.A. 1991, p. 44. 3 ZUMTHOR, Peter. Atmósferas: entornos arquitectónicos, las cosas a mi
alrededor. Introduccióna la charla. Barcelona: Gustavo Gili, cop. 2006. 4 Término acuñado por primera vez por Manfredo Tafuri en Teorías e histo-
ria de la arcuitectura, 1997, pero analizado anteriormente en autores como
Rudolf Wittitkower en Los fundamentos de la arquitectura en la edad del hu-

${ }^{5}$ Método utilizado desde el siglo XIX en la enseñanza de la composición

6 Para un análisis magistral del Arte de la fuga de Bach véanse los estudios
de Donald Tovey. 7 NORBERG-SCHUL7, Christian, Intenciones en arquitectura, Gustavo Gili,
1979, Barcelona, p. 100. 8 SCHOENBERG, Arnold, Fundamentos de la composición musical, Real
Musical, Madrid, 1979. 9 BACHELARD, Gaston,
de España, 2000, p. 44.

STRAVINSKY, Igor. Poética musical, Taurus ediciones, Madrid 1981,p. 33. Se recomienda escuchar el fragmento de la Danza de la tierra en:
tp://Circuitodearquitectura.org/caleidoscopio/mus_stravinski/

12 Me refiero a un análisis fenomenológico amplio, no particularmente al con-
cepto ofilosófico de la fenomenologia que Edmund Husserl instaura, aunque tiene allí sus ráices. Para una visión reciente de la fenomenología en la arquitectu-
ra véanse los ensayos de Juahanni Pallasmaa, en particular Los ojos de la piel. 13 Véase MONTANER, Josep Maria, Sistemas arquitectónicos contemporá-
neos, Gustavo Gili, Barcelona, 2009. ${ }^{14}$ Véase VENTURI, Robert, Complejidad y contradicción en la arquitectura,
Gustavo Gili, Barcelona, 1978. ${ }^{15} \mathrm{Se}$ recomienda escuchar el inicio del preludio en: http://circuitodearquitec-
tura.org/caleidoscopio/mus debussy/mus debussy.html ${ }^{16}$ GUARDINI, Romano, "Sobre la esencia de la obra de arte", en Imagen de
culto imagen de devocionn, Sobre la esencia de la obra de arte, Madrid,
Guadarrama, 1960, p. 68 .

BIBLIOGRAFIA

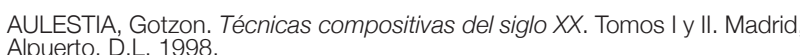
BACHELARD, Gas

ESPAÑOL, Joaquim. El orden frágil de la arquitectura. Barcelona: Fundación GUARDINI, Romano, "Sobre la esencia de la obra de arte", en Imagen de
culto e imagen de devoción; Sobre la esencia de la obra de arte, Madrid, MONTANER, Josep Maria, Sistemas arquitectónicos contemporáneos, GusNORBERG-SCHULZ, Christian, Intenciones en arquitectura, Gustavo Gili,
1979, Barcelona, p. 100. PALLASMAA, Juhani. Los ojos de la piel: la arquitectura y los sentidos. Bar-
celona: Gustavo Gili, cop. 2006. ROSS, Alex. El ruido eterno: escuchar al siglo XX a través de su música, Trad.
De Luis Gago, Barcelona, Seix Barral, 2012 . SCHOENBERG Arnold, Fundamentos de la composición musical, Real Mu-
sical, Madrid, 1979 . STRAVINSKY, Ígor. Poética musical, Taurus ediciones, Madrid 1981. TRÍAS, Eugenio, Lógica del límite. Eugenio Trías, Ediciones Destino, S.A. 1991. VENTURI, Robert, Complejidad y contradicción en la arquitectura, Gustavo
Gili, Barcelona 1978. ZUMTHOR, Peter. Atmósferas: entornos arquitectónicos, las cosas a mi alre-
dedor. Barcelona: Gustavo Gili, cop. 2006.

Josep Llorca es estudiante de tercer curso en la E.T.S.A.Barcelona. 